九州大学学術情報リポジトリ

Kyushu University Institutional Repository

\title{
GENERALIZATION OF NEYMAN-PEARSON FUNDAMENTAL LEMMA TO VECTOR-VALUED DECISION FUNCTIONS AND ITS APPLICATION TO UNBIASED SLIPPAGE TESTS
}

Yeh, Neng-Che

Tamkang College of Arts and Sciences | Kyushu University

https://doi.org/10.5109/13032

出版情報：統計数理研究. 13 (1/2), pp.73-83，1968-03. Research Association of Statistical Sciences

バージョン :

権利関係 : 


\title{
GENERALIZATION OF NEYMAN-PEARSON FUNDAMENTAL LEMMA TO VECTOR-VALUED DECISION FUNCTIONS AND ITS APPLICATION TO UNBIASED SLIPPAGE TESTS
}

\author{
By \\ Neng-che YEH \\ (Received January 30th, 1968)
}

\section{$\S 1$. Introduction.}

Slippage problems have been discussed by various authors such as Mostellar [7], Paulson [9], Traux [10], Doornbos and Prins [1], Kudô [5], Karlin and Traux [4], and others.

In particular a class of slippage problems has been mainly treated in a manner analogous to the treatment of problems in hypotheses testing (Lehmann [9]). In general, the problem is as follows. Assuming that we have $a$ populations with densities $p\left(x ; \theta_{i}\right)(i=1, \cdots, a)$ and we wish to test the hypothesis $H_{0}: \theta_{1}=\cdots=\theta_{a}$ against $a$ alternatives $H_{i}: \theta_{1}=\cdots=\theta_{i}-\Delta=\cdots=\theta_{a}$ with a zero-one type loss function, where $\Delta>0$.

Hall and Kudô [2] gave a generalization of Neyman-Pearson lemma so as to be useful in solving slippage problems where the null hypothesis is simple and the alternatives are simple. They also applied their generalized lemma to slippage problems where the alternatives are composite for the population distribution for which satisfying certain conditions closely connected with monotone likelihood ratio. The existence of an uniformly most powerful test under some conditions was also given. Furthermore, Hall, Kudô and Yeh [3] treated a similar test for the slippage problem.

In this paper we shall start with a new generalization of Neyman-Peason lemma with respect to vector-valued decision function which will be given in Theorem 1 in section 2. This Theorem 1 is crucially important for establishing unbiased slippage test as we shall show in section 3. In view of these results the author proceed to give another generalization of Neyman-Peason lemma which will be useful in treating a certain type of testing hypothesis whose formulation is given in section 4 . This certain type of testing hypothesis is: under the hypothesis $H_{0}$, a random variable $X$ has a density equal to one of the densities $h_{i}(x)(i=1, \cdots, k)$, while each of its alternatives $H_{i}$ has the density $h_{k+i}(x)(i=1, \cdots, a)$. For the particular case, i. e. $a=k$, is given in section 5 . 


\section{$\S 2$. A generalization of the fundamental lemma to vector-valued decision functions (I).}

Let $\mathfrak{X}$ be a Enclidean space and $\mathfrak{T}$ be a $\sigma$-field of subsets of $\mathfrak{X}$ with a measure $\mu$ on $\mathfrak{i}$. Let $f_{1}, f_{2}, \cdots, f_{a}, h_{0}, h_{1}, \cdots, h_{a}$ be real-valued functions defined on the space $X$ and integrable $\mu$. Suppose that for a given constants $c_{0}$ and $c_{i}(i=1,2, \cdots, a)$ there exists a vector-valued decision function $\varphi(x)=\left(\varphi_{0}(x), \varphi_{1}(x), \cdots, \varphi_{a}(x)\right)$ with $\sum_{i=0}^{a} \varphi_{i}(x)=1$, $0 \leqq \varphi_{i} \leqq 1$ for $i=0,1, \cdots, a$, satisfying

$$
\int \varphi_{0}(x) h_{0}(x) d \mu(x)=c_{0} \text { and } \int \varphi_{i}(x) f_{i}(x) d \mu(x)=c_{i}
$$

for $i=1,2, \cdots, a$.

Denote by $C$ the class of vector-valued decision functions $\varphi$ for which (2.1) holds. Unless otherwise explicitly expressed, all integrals $\int$ considered in the present and following sections are $\int_{x}$. Then we have the following theorem.

Theorem 1.

(i) Existence. $\operatorname{Max}_{\varphi \in C}\left[\sum_{i=1}^{a} \int \varphi_{i}(x) h_{i}(x) d \mu(x)\right]$ exists.

(ii) Sufficiency. If a decision function $\varphi$ in $C$ satisfies

$$
\begin{aligned}
& \varphi_{0}(x)=1, \xi(x), 0 \quad \text { when } \quad k_{0} h_{0}>,=,<\max _{i}\left[\left(k_{i} f_{i}+h_{i}\right)\right], \\
& \varphi_{j}(x)=\eta_{j}(x), 0 \quad \text { when } \quad k_{j} f_{j}+h_{j}=,<\max _{i}\left[\left(k_{i} f_{i}+h_{i}\right)\right],
\end{aligned}
$$

where $\xi(x)$ and $\eta_{j}(x)$ are arbitrary, subject to the decision function $\varphi$, for some $k_{0}$ and $k_{i}(i=1,2, \cdots, a)$, then this $\varphi$ maximizes

$$
\sum_{i=1}^{a} \int \varphi_{i}(x) h_{i}(x) d \mu(x)
$$

among $C$.

(iii) The set $D$ of points in $(a+1)$-dimensional space whose coordinates are $\left(\int \varphi_{0} h_{0} d \mu, \int \varphi_{1} f_{1} d \mu, \cdots, \int \varphi_{a} f_{a} d \mu\right)$ for some decision function $\varphi$ is convex and closed.

(iv) Necessity. A decision function in $C$ satisfying (2.2) is attainable, provided $\left(c_{0}, c_{1}, \cdots, c_{a}\right)$ is an inner point of $D$, and if a member of $C$ maximizes (2.3) then for some $k_{0}$ and $k_{i}$, it satisfies (2.2) a.e. $\mu$.

Proof. (i) Consider a sequence $\left\{\varphi^{(n)}(x)\right\}$ of the set $C$ such that $\sum_{i=1}^{a} \int \varphi_{i}^{(n)}(x) h_{i}(x) d \mu(x)$ tends to $\operatorname{Sup}_{\varphi \equiv C}\left[\sum_{i=1}^{a} \int \varphi_{i}(x) h_{i}(x) d \mu(x)\right]$. By the generalization of the weak compactness theorem to vector-valued functions (Kudô [6]), there is a subsequence $\left\{\varphi^{\left(n_{j}\right)}(x)\right\}$ of $\left\{\varphi^{(n)}(x)\right\}$ and $\varphi^{0}(x)$ such that

$$
\int \varphi_{i}^{\left(n_{j}\right)} f(x) d \mu(x) \stackrel{n_{j}}{\longrightarrow} \int \varphi_{i}^{(0)} f(x) d \mu(x) \quad(i=0,1, \cdots, a)
$$

for all integrable function $f(x)$ with respect to $\mu$. Since 


$$
\int \varphi_{0}^{\left(n_{j}\right)}(x) h_{0}(x) d \mu(x) \stackrel{n_{j}}{\longrightarrow} \int \varphi_{0}^{(0)}(x) h_{0}(x) d \mu(x)
$$

$O^{(x)}(x)$ belongs to $C$. On the other hand,

$$
\sum_{i=1}^{a} \int \varphi_{i}^{\left(n_{j}\right)}(x) h_{i}(x) d \mu(x) \stackrel{n_{j}}{\longrightarrow} \sum_{i=1}^{n} \int \varphi_{i}^{(0)}(x) h_{i}(x) d \mu(x) .
$$

By the uniqueness,

$$
\sum_{i=1}^{a} \int \varphi_{i}^{\prime \prime}(x) h_{i}(x) d \mu(x)=\operatorname{Sup}_{\varphi \in C}\left[\sum_{i=1}^{a} \int \varphi_{i}(x) h_{i}(x) d \mu(x)\right] .
$$

Hence $\operatorname{Max}_{\varphi \Subset O}\left[\sum_{i=1}^{a} \int o_{i}(x) h_{i}(x) d \mu(x)\right]$ exists.

(ii) Let $\varphi$ in $C$ satisfy (2.2) and $\hat{\varphi}$ be any other one in $C$. We consider

$$
\sum_{i=1}^{a} \int \varphi_{i}(x) h_{i}(x) d \mu(x)-\sum_{i=1}^{a} \int \hat{\varphi}_{i}(x) h_{i}(x) d \mu(x) \equiv \int g(x) d \mu(x),
$$

where

$$
g(x)=\left[\varphi_{0} k_{0} h_{0}+\sum_{i=1}^{a} \varphi_{i}\left(k_{i} f_{i}+h_{i}\right)\right]-\left[\hat{\varphi}_{0} k_{0} h_{0}+\sum_{i=1}^{a} \hat{\varphi}_{i}\left(k_{i} f_{i}+h_{i}\right)\right] .
$$

We devide the sample space $\mathfrak{x}$ into three disjoints regions:

$$
\left\{x: \varphi_{0}(x)=1\right\},\left\{x: \varphi_{0}(x)=\xi(x)\right\} \text { and }\left\{x: \varphi_{0}(x)=0\right\} .
$$

We shall verify that the $g(x) \geqq 0$ in each of these regions.

For $\left\{x: \varphi_{0}(x)=1\right\}$, we have

$$
g(x) \geqq\left(1-\hat{\varphi}_{0}\right) k_{0} h_{0}-\max _{i}\left(k_{i} f_{i}+h_{i}\right) \sum_{i=1}^{a} \hat{\varphi}_{i}>\left(1-\hat{\varphi}_{0}\right) k_{0} f_{0}-k_{0} h_{0} \sum_{i=1}^{a} \hat{\varphi}_{i}=0,
$$

for $\left\{x: \varphi_{0}(x)=\xi(x)\right\}$,

$$
g(x) \geqq\left(\xi-\hat{\varphi}_{0}\right) k_{0} h_{0} \div \sum_{i=1}^{a} \varphi_{i}\left(k_{i} f_{i}+h_{i}\right)-\max _{i}\left(k_{i} f_{i}+h_{i}\right) \sum_{i=1}^{a} \hat{\varphi}_{i}=k_{0} h_{0}-k_{0} h_{0} \sum_{i=1}^{a} \hat{\varphi}_{i}=0 .
$$

And by the same argument we have $g(x) \geqq 0$ for $x$ in $\left\{x: \varphi_{0}(x)=0\right\}$. Therefore

$$
\sum_{i=0}^{a} \int \varphi_{i}(x) h_{i}(x) d \mu(x) \geqq \sum_{i=1}^{a} \int \hat{\varphi}_{i}(x) h_{i}(x) d \mu(x) .
$$

The assertion of (ii) is thus proved.

(iii) The closedness of the set $D$ follows from the generalization of the weak compactness theorem to vector-valued decision functions and the convexity of the set $D$ follows from

$$
\sum_{i=0}^{a}\left[\lambda \varphi_{i}+(1-\lambda) \hat{\varphi}_{i}\right]=1, \quad 0 \leqq \lambda \leqq 1 .
$$

We note that the set $E$ of points in $(a+2)$-dimensional space whose coordinates are $\left(\int \varphi_{0} h_{0} d \mu, \int \varphi_{1} f_{1} d \mu, \cdots, \int \varphi_{a} f_{a} d \mu, \int \sum_{i=1}^{a} \varphi_{i} h_{i} d \mu\right)$ for some decision function is also convex and closed. The points of $E$, the first $(a+1)$ coordinates of which are $c_{0}, c_{1}$, $\cdots, c_{a}$, form a closed interval $\left[c^{*}, c^{* *}\right]$. Since $\left(c_{0}, c_{1}, \cdots, c_{a}, c^{* *}\right)$ is a boundary point of $E$, there is a hyperplane through it such that every point of $E$ lies below or on this hyperplane. Let us write the equation of the hyperplane

$$
k_{0} \int \varphi_{0} h_{0} d \mu+\sum_{i=1}^{a} k_{i} \int \varphi_{i} f_{i} d \mu+k_{a+1} \int \sum_{i=1}^{a} \varphi_{i} h_{i} d \mu=\sum_{i=0}^{a} k_{i} c_{i}+k_{a+1} c^{* *} .
$$


Since $\left(c_{0}, c_{1}, \cdots, c_{a}\right)$ is an inner point of $D$, the $k_{a \div 1} \neq 0$ and

$$
\int\left[\sum_{i=1}^{a}\left(h_{i}+k_{i} f_{i}\right) \varphi_{i}+k_{0} h_{0} \varphi_{0}\right] d \mu \leqq \int\left[\sum_{i=1}^{n}\left(h_{i}+k_{i} f_{i}\right) \varphi_{i}^{* *}+k_{0} h_{0} \varphi_{0}^{* *}\right] d \mu
$$

for all points in $E$, where $\varphi^{* *}$ is the decision function given rise to the point $\left(c_{0}, c_{1}\right.$, $\left.\cdots, c_{a}, c^{* *}\right)$. Thus $\varphi^{* *}$ maximizes the left-hand side of (2.10). Since the integral is maximized by putting $\varphi$ as the form of (2.2), $\varphi^{* *}$ satisfies (2.2) a. e. $\mu$ the assertions (iii) and (iv) are thus proved.

In the present section we restrict $h_{i}(x)(i=0,1, \cdots, a)$ to be probability density of $X$ with respect to $\mu$, and denote $\int \varphi_{i}(x) h_{i}(x) d \mu(x)$ by $E_{i} \varphi_{i}(X)(i=0,1, \cdots, a)$.

Definition 1. A decision function $\varphi(x)=\left(\varphi_{0}(x), \varphi_{1}(x), \cdots, \varphi_{a}(x)\right)$ is of size $\alpha$ if

$$
E_{0} \varphi_{0}(X) \geqq 1-\alpha \text {. }
$$

It is of exact size $\alpha$ if the equality holds.

DEFINITION 2. $\varphi(x)$ is symmetric in power if

$$
E_{1} \varphi_{1}(X)=\cdots=E_{a} \varphi_{a}(X) .
$$

The common value of (2.12) is called the power of $\varphi$. It is called the most powerful symmetric of size $\alpha$ (MPSS $\alpha$ ) if it maximizes each term of (2.12) subject to (2.11) and (2.12).

Throughout this paper, we consider a measurable transformation group $G$ on $\mathfrak{x}$, which is isomorphic to the permutation group, $\Pi=\left\{\pi_{g}\right\}$ on $(1,2, \cdots, a)$ or its transitive subgroup.

Definition 3. $\varphi(x)$ is invariant under the group $G=\{g\}$ if

$$
\varphi_{0}(x)=\varphi_{0}(g x) \text { and } \varphi_{i}(x)=\varphi_{\pi_{g}}(g x) \quad \text { for all } g \text { and } i,
$$

where $\pi_{g}$ is a permutation on $(1,2, \cdots, a)$ corresponding to $g$.

Now we assume that there is a measurable transformation group $G$ on $\stackrel{t}{*}$, which is isomorphic to the permutation group, $\pi$, or to its transitive subgroup, such that $f_{i}(x)=f_{\pi_{g i}}(g x), h_{i}(x)=h_{\pi_{g i}}(g x)$ and $h_{0}(x)=h_{0}(g x)$ for all $g$ and $i$, and $\mu(A)=\mu(g A)$ for all $A \in \mathfrak{A}$ and $g$. Then the following Corollary follows.

COROLLARY 1. Consider the class of decision functions satisfying

$$
E_{0} \varphi_{0}(X)=1-\alpha \alpha E(0,1) \text { and } \int \varphi_{i}(x) f_{i}(x) d \mu(x)=c \text { for all } i .
$$

Let $C_{\alpha}$ be the class of $\varphi$ for which (2.14) holds.

(i) Given any $\alpha$, the most powerful symmetric of size $\alpha$ (MPSS $\alpha$ ) decision function exists among $C_{\alpha}$, furthermore, it is invariant.

(ii) If $\varphi \in C_{\alpha}$ satisfies

$$
\varphi_{0}(x)=1, \xi, 0 \quad \text { when } k_{0} h_{0}>,=,<\max _{i}\left[\left(k f_{i}+h_{i}\right)\right],
$$

$$
\varphi_{j}(x)=\frac{1-\varphi_{0}(x)}{J(x)} 0, \text { when } k f_{j}+h_{j}==,<\max _{i}\left[\left(k f_{i}+h_{i}\right)\right],
$$

where $J(x)$ is the number of times that $\max \left[\left(k f_{i}+h_{i}\right)\right]$ is attained, for some $k_{0}$ and $k$, then it is a MPSS $\alpha$ decision function among $C_{\alpha}$. 
(iii) $k_{0}$ and $k$ in (ii) is attainable for $\varphi \in C_{\alpha}$ if the point $(1-\alpha, c, \cdots, c)$ is an inner point of the set $D$, and if $\varphi$ is MPSS $\alpha$ among $C_{\alpha}$ then it satisfies (2.15) a.e. $\mu$.

Proof. (i) $\mathrm{By}$ (i) of Theorem 1, $\operatorname{Max}_{\varphi \in C_{\alpha}}\left[\sum_{i=1}^{n} E_{i} \varphi_{i}(X)\right]$ exists, say, $\hat{\varphi}(x)$. As the group $G$ is finite, there is a right invariant probability measure $\nu$ on $(G, \beta)$ measurable space, where $\beta$ is a $\sigma$-field of subsets of $G$. We may define $\varphi$ as

$$
\hat{\hat{\varphi}}_{i}(x)=\int \hat{\varphi}_{\pi_{g} i}(g x) d \nu(g) \quad i=0,1, \cdots, a .
$$

This $\hat{\hat{\varphi}}$ is invariant and belongs $C_{a}$. We see that

$$
\begin{aligned}
\sum_{i=1}^{a} E_{i} \hat{\varphi}_{i}(X) & =\sum_{i=1}^{a} \iint \hat{\varphi}_{\pi_{g} i}(g x) h_{i}(x) d \mu(x) d \nu(g) \\
& =\int\left[\sum_{i=1}^{a} \int \hat{\varphi}_{\pi_{g} i}(g x) h_{\pi_{g}}(g x) d \mu(g x)\right] d \nu(g) \\
& =\int\left[\sum_{i=1}^{a} E_{\pi_{g i}} \hat{\varphi}_{\pi_{g}}(X)\right] d \nu(g) \\
& =\int\left[\sum_{i=1}^{a} E_{i} \hat{\varphi}_{i}(X)\right] d \nu(g)=\sum_{i=1}^{a} E_{i} \hat{\varphi}_{i}(X) .
\end{aligned}
$$

Hence $\hat{\hat{\varphi}}$ is MPSS $\alpha$ among $C_{\alpha}$.

(ii) The proof is analogous to that of (ii) of Theorem 1, we shall omit it.

(iii) By (iii) of Theorem 1, there are $k_{0}, k_{1}, \cdots, k_{a}$. We note that the (2.10) of Theorem 1 implies

$$
\begin{aligned}
& \int\left[\sum_{i=1}^{a}\left(h_{\pi_{g} i}+k_{i} f_{\pi_{g} i}\right) \varphi_{\pi_{g} i}+k_{0} h_{0} \varphi_{0}\right] d \mu \\
& \quad \leqq \int\left[\sum_{i=1}^{a}\left(h_{i}+k_{i} f_{i}\right) \varphi_{i}^{* *}+k_{0} h_{0} \varphi_{0}^{* *}\right] d \mu \quad \text { for all } g \in G .
\end{aligned}
$$

We can see this by putting $\phi_{i}\left(g^{-1} x\right)=\varphi_{\pi_{g} i}(x)$ and $\left.\phi_{0}\left(g^{-1} x\right)\right)=\varphi_{0}(x)$. Then by (ii) we have

$$
\begin{aligned}
\int\left[\sum_{i=1}^{a}\left(h_{i}+\frac{k_{1}+\cdots+k_{a}}{a} f_{i}\right) \varphi_{i}+k_{0} h_{0} \varphi_{0}\right] d \mu \\
\leqq \int\left[\sum_{i=1}^{a}\left(h_{i}+k_{i} f_{i}\right) \varphi_{i}^{* *}+k_{0} h_{0} \varphi_{0}^{* *}\right] d \mu .
\end{aligned}
$$

Thus the assertion is proved by putting $\frac{k_{1}+\cdots+k_{a}}{a}=k$.

\section{§3. Unbiasedness on slippage test.}

Let $\theta$ be a parameter in a parameter space $\Omega$ and $X$ a random variable with probability density $p_{\theta}(x)$ with respect to a $\sigma$-additive measure $\mu$ on a $\sigma$-field of subsets of a sample space $\mathfrak{X}$. Suppose the parameter space is divided into $a+1$ disjoint subsets $\Omega_{0}, \Omega_{1}, \cdots, \Omega_{a}$. We shall consider a decision problem involving $a+1$ possible decisions denoted by $D_{i}(i=0,1, \cdots, a)$ corresponding to $a+1$ hypotheses $H_{0}: \theta \in \Omega_{0}$, 
$H_{1}: \theta \in \Omega_{1}, \cdots, H_{a}: \theta \in \Omega_{a}$, respectively, where under $H_{0}, X$ has density equal $p_{\theta}(x)$, $\theta \in \Omega_{0}$ and under $H_{i}$ the density $p_{\theta}(x), \theta \in \Omega_{i}$ for $i=1, \cdots, a$. The vector-valued decision functions of the form $\varphi(x)=\left(\varphi_{0}(x), \varphi_{1}(x), \cdots, \varphi_{a}(x)\right)$ with $\varphi_{i}(x)$ denotes the probability of marking $D_{i}$ for $i=0,1, \cdots, a$, as the random variable $X$ taking value $x$, are considered. Of course we have

$$
\sum_{i=0}^{a} \varphi_{i}(x)=1, \quad 0 \leqq \varphi_{i} \leqq 1 .
$$

Because of similarity to the theory of a testing hypothesis, we shall call $H_{0}$ the null hypothesis, $H_{i}(i=1, \cdots, a)$ the alternative, and this problem is called hereafter a test of $H_{0}$ against $H_{i}$ for $i=1, \cdots, a$.

Definition. A decision function $\varphi(x)$ is unbiased of size $\alpha$ if

$$
E_{\theta_{0}} \varphi_{1}(X)=\cdots=E_{\theta_{0}} \varphi_{a}(X), \sum_{i=1}^{a} E_{\theta_{0}} \varphi_{i}(X) \leqq \alpha \quad \text { for } \quad \theta_{0} \in \Omega_{0}
$$

and

$$
\sum_{i=1}^{a} E_{\ell_{i}} \varphi_{i}(X) \geqq \alpha \text { for } \quad \theta_{i} \in \Omega_{i}, \quad i=1, \cdots, a .
$$

Let $X$ be a random variable with probability density

$$
p_{6}(x)=C(\theta) \exp \left[\theta^{\prime} T(x)\right]
$$

with respect to $\mu$, where $\theta$ and $T(x)$ both are the points in $a$-dimensional Euclidean space, i. e. $\theta^{\prime}=\left(\theta^{(1)}, \theta^{(2)}, \cdots, \theta^{(a)}\right)$ and $T^{\prime}(x)=\left(T_{1}(x), \cdots, T_{a}(x)\right)$. Assume that the parameter space is divided into a curves and a point which are given by $\theta=\theta_{i}(\tau)$, $-\infty<\tau<\infty, i=1, \cdots, a$ and a common starting point $\theta_{0}=\theta_{i}(0)$. We also assume that there are transformation groups $G=\{g\}$ and $\Pi=\left\{\pi_{g}\right\}$ as stated in the previous section such that $p_{\theta_{0}}(x)=p_{\theta_{0}}(g x), p_{\theta_{i}(\tau)}(x)=p_{\theta_{\pi_{g} i}(\tau)}(g x)$ and $T_{i}(x)=T_{\pi_{g} i}(g x)$ for all $i, \tau$, $g \in G$. Then we have the following theorem.

THEOREM 2. Let $\theta_{i}(\tau)=\left(\theta^{(0)}, \theta^{(0)}, \cdots, \theta^{(0)}+\tau, \theta^{(0)}, \cdots, \theta^{(0)}\right)$, where $\theta^{(0)}+\tau$ is the $i$-th coordinate of the point $\theta_{i}(\tau)$, and $-\infty<\tau<\infty$. Suppose $C\left(\theta_{i}(\tau)\right)$ depends upon $\tau$ only. Then the following $\varphi$ constitutes a MPSS $\alpha$ unbiased test uuiformly in $\tau$ for testing

$$
H_{0}: \theta=\theta_{0}=\theta_{i}(0)(\text { known })
$$

against

$$
H_{i}: \theta=\theta_{i}(\tau), \quad-\infty<\tau<\infty, \quad \tau \neq 0 \quad i=1, \cdots, a .
$$

The $\varphi$ is given by

$$
\begin{aligned}
& \varphi_{0}(x)= \text { when both } \min _{i} T_{i}>K_{0} \text { and } \max _{i} T_{i}<K, K_{0}<K \\
&= 0 \text { when } \min _{i} T_{i}<K_{0} \text { or } \max _{i} T_{i}>K \\
&= \text { when both } \min _{i} T_{i}=K_{0} \text { and } \max _{i} T_{i} \leqq K \text { or both } \\
& \qquad \min _{i} T_{i} \geqq K_{0} \text { and } \max _{i} T_{i}=K \\
& \varphi_{j}(x)=\frac{1-\varphi_{0}(x)}{J(x)} \text { when } T_{j}=\max _{i} T_{i} \geqq K \text { or } T_{j}=\min _{i} T_{i} \leqq K_{0} \\
&=0, \quad \text { otherwise, }
\end{aligned}
$$


where $J(x)$ is the number of times that $\max T_{i}$, in addition $\min T_{i}$ are attained. $K$ 's and $\xi$ are determined by

$$
E_{\theta_{0}} \varphi_{0}(X)=1-\alpha \text { and } E_{\theta_{0}}\left[T_{i}(X) \varphi_{i}(X)\right]=-\frac{\alpha}{a} E_{\theta_{0}}\left[T_{i}(X)\right] \text { for all } i .
$$

Proof. By Theorem 9 of Chap. 2 in Lehman [8], $E_{t_{i}} \varphi_{i}(X)$ is analytic function of $\theta^{(j)}(j=1, \cdots, a)$, where $\theta^{(j)}$ is the $j$-th coordinate of the vector $\theta_{i}$ therefore unbiasedness implies

$$
E_{\theta_{0}} \varphi_{0}(X)=1-\alpha \text { and } E_{\theta_{0}}\left[T_{i}(X) \varphi_{i}(X)\right]=E_{\theta_{0}}\left[\varphi_{i}(X)\right] E_{\theta_{0}}\left[T_{i}(X)\right] \text { for all } i \text {. }
$$

We note that

$$
E_{\theta_{0}}\left[T_{i}(X)\right]=E_{\theta_{\pi_{g} 0}}\left[T_{\pi_{g i}}(g x)\right]=E_{\theta_{0}}\left[T_{j}(x)\right] \quad \text { and } \quad E_{0_{0}}\left[\varphi_{i}(X)\right]=E_{\theta_{0}}\left[\varphi_{i}(X)\right] .
$$

Hence, by the corollary 1 in $\S 2$, there is a MPSS $\alpha$ test for each $\tau$, given by

$$
\begin{aligned}
\varphi_{0}(x) & =1, \xi, 0 \text { when } k_{0}(\tau) p_{\theta_{0}}(x)>,=,<\max _{i}\left[k(\tau) T_{i}(x) p_{\theta_{0}}(x)+p_{\theta_{i}}(x)\right], \\
\varphi_{j}(x) & =\frac{1-\varphi_{0}(x)}{J(x)}, 0 \\
& \text { when } k(\tau) T_{j}(x) p_{\theta_{0}}(x)+p_{\theta_{j}}(x)=,<\max _{i}\left[k(\tau) T_{i}(x) p_{\theta_{0}}(x)+p_{\theta_{i}}(x)\right] .
\end{aligned}
$$

We note that $p_{\theta_{i}(\tau)}(x)=\frac{c\left(\theta_{i}(\tau)\right)}{c\left(\theta_{0}\right)} p_{\theta_{0}}(x) e^{\tau \tau_{i}(x)}$, then (3.4) immediately follows.

Example. Let $X=\left(X_{1}, X_{2}\right)$ be distributed according to the bivariate normal distribution with mean $\theta=\left(\theta^{(1)}, \theta^{(2)}\right)$ and covariance matrix I. Suppose the parameter space is $\Omega=\{(0,0),(\tau, 0),(0, \tau) ; \tau \neq 0\}$. Then a most powerful symmetric of size $\alpha$ unbiased test uniformly in $\tau$ is given by

$$
\begin{aligned}
\varphi_{0}(x) & =1 \quad \text { when }-c<x_{1}, x_{2}<c \\
& =0 \quad \text { when } \min \left(x_{1}, x_{2}\right)<-c \text { or } \max \left(x_{1}, x_{2}\right)>c \\
& =\xi \quad \text { when }\left(x_{1}, x_{2}\right) \text { is on the side of the square with center } \\
& \quad \text { at the origin and side length } 2 c . \\
\varphi_{j}(x) & =\frac{1-\varphi_{0}(x)}{J(x)} \quad \text { when } x_{j}=\max \left(x_{1}, x_{2}\right) \geqq c \text { or } x_{j}=\min \left(x_{1}, x_{2}\right) \leqq-c \\
& =0, \quad \text { otherwise. }
\end{aligned}
$$

$\therefore$ is chosen such that $p_{r}\left[-c<x_{1}, x_{2}<c \mid(0,0)\right]=1-\alpha$.

\section{§4. A generalization of the fundamental lemma to vector-valued decision functions (II).}

Let $\mathfrak{X}$ be a sample space and let $\mathfrak{X}$ be a $\sigma$-field of subsets of $\mathfrak{X}$ with a countable number of generatores. Suppose we have $k+a$ densities $h_{i}(x), i=1, \cdots, k, k+1$, $\cdots, k+a$, with respect to a $\sigma$-additive measure $\mu$ on $\mathfrak{i}$. We shall consider a decision problem involving $a+1$ decisions $D_{0}, D_{1}, \cdots, D_{a}$ corresponding to $a+1$ hypotheses $H_{0}, H_{1}, \cdots, H_{a}$, respectively, where under $H_{0}, X$ has a density equal to one of the $h_{i}(x)(i=1,2, \cdots, k)$ and under $H_{i}$ the density $h_{k+i}(x)(i=1,2, \cdots, a)$. We shall denote by $E_{i}($,$) the expectation of ($,$) when the X$ have probability density $h_{i}(x)$. 
THEOREM 3. Let $C_{0}=\left\{\varphi(x) ; E_{i} \varphi_{0}(X) \geqq c_{0}, i=1, \cdots, k\right\}$.

(i) $\operatorname{Sup}_{\varphi=C_{0}} \sum_{i=1}^{a} E_{k+i} \varphi_{i}(X)$ is attained by some one in $C_{0}$.

(ii) If a decision function $\varphi(x)$ satisfies $E_{i} \varphi_{0}(X)=c_{0}$ for $i=1, \cdots, k$ with the form

$$
\begin{aligned}
& \varphi_{0}(x)=1, \xi(x), 0 \text { when } \max _{i=1, \cdots, a} h_{k+i}(x)<,=,>M_{0^{-}}^{1} \cdot \sum_{i^{\prime}=1}^{k} h_{i^{\prime}}(x) \\
& \varphi_{j}(x)=\eta_{j}(x), 0 \quad \text { when } h_{k+j}(x)=,<\max _{i=1, \cdots, a} h_{k+i}(x),
\end{aligned}
$$

where $\xi(x)$ and $\eta_{j}(x)$ are arbitrary, subject to the decision function $\varphi$ and $M_{0}(\geqq 0)$ is a constant.

Then

$$
\sum_{i=1}^{a} E_{k+i} \varphi_{i}(X) \geqq \sum_{i=1}^{a} E_{k+i} \hat{\varphi}_{i}(X)
$$

for any other $\hat{\varphi}(x)$ in $C_{0}$.

Proof. (i) Consider a sequence $\left\{\varphi^{(n)}(x)\right\}$ of the set $C_{0}$ such that $\sum_{i=1}^{a} E_{k+i} \varphi_{i}^{(n)}(X)$ tends to $\operatorname{Sup}_{\varphi \in C_{0}} \sum_{i=1}^{a} E_{k \div i} \varphi_{i}(X)$. By the generalization of the weak compactness theorem to vector-valued functions (Kudô [6]), there is a subsequence $\left\{\varphi^{\left(n_{j}\right)}(x)\right\}$ of $\left\{\varphi^{(n)}(x)\right\}$ and $\varphi^{(0)}(x)$ such that

$$
\int \varphi_{i}^{\left(n_{j}\right)} f(x) d \mu(x) \rightarrow \int \varphi_{i}^{(0)} f(x) d \mu(x)
$$

for all integrable $f(x)$ w.r.t. $\mu$. Since

$$
\int \varphi_{0}^{\left(n_{j}\right)}(x) h_{i}(x) d \mu(x) \rightarrow \int \varphi_{0}^{(0)} h_{i}(x) d \mu(x) \quad \text { for } \quad i=1, \cdots, k,
$$

$\varphi^{(0)}(x)$ belongs to $C_{0}$. On the other hand,

$$
\sum_{i=1}^{a} E_{k+i} \varphi^{\left(n_{j}\right)}(X) \rightarrow \operatorname{Sup}_{\varphi \sqsubseteq C_{0}} \sum_{i=1}^{a} E_{k+i} \varphi_{i}(X) .
$$

Hence $\varphi^{(0)}(x)$ maximizes $\sum_{i=1}^{a} E_{k+i} \varphi_{i}(X)$ among $C_{0}$.

(ii) Consider the integral $\int g(x) d \mu(x)$, where

$$
g(x)=\left(\varphi_{0}(x)-\hat{\varphi}_{0}(x)\right) M_{0} \cdot \frac{1}{k} \cdot \sum_{i=1}^{k} h_{i}(x)+\sum_{i=1}^{a}\left(\varphi_{i}(x)-\hat{\varphi}_{i}(x)\right) h_{k+i}(x),
$$

$\varphi(x)$ satisfies $E_{i} \varphi_{0}(x)=c_{0}(i=1, \cdots, k)$ with the form (4.1) and $\hat{\varphi}(x)$ is any other decision function in $C_{0}$.

We devide the sample space $\mathfrak{X}$ into there disjoint regions: $\left\{x: \varphi_{0}(x)=1\right\},\left\{x: \varphi_{0}(x)\right.$ $=\xi(x)\}$ and $\left\{x: \varphi_{0}(x)=0\right\}$. It will be verified that $g(x) \geqq 0$ in each of these regions. For $\left\{x: \varphi_{0}(x)=1\right\}$,

$$
\begin{aligned}
g(x) & =\left(1-\hat{\varphi}_{0}(x)\right) M_{0} \cdot \frac{1}{k} \cdot \sum_{i^{\prime}=1}^{k} h_{i^{\prime}}(x)-\sum_{i=1}^{a} \hat{\varphi}_{i}(x) h_{k+i}(x) \\
& \geqq\left(1-\hat{\varphi}_{0}(x)\right)\left(M_{0} \cdot \frac{1}{k} \sum_{i^{\prime}=1}^{k} h_{i^{\prime}}(x)-M_{0} \cdot \frac{1}{k}-\sum_{i^{\prime}=1}^{k} h_{i^{\prime}}(x)\right)=0,
\end{aligned}
$$

for $\left\{x: \varphi_{0}(x)=\xi(x)\right\}$, 


$$
\begin{aligned}
g(x) & \geqq\left(\varphi_{0}(x)-\hat{\varphi}_{0}(x)\right) M_{0} \cdot 1 \frac{1}{k} \sum_{i^{\prime}=1}^{k} h_{i^{\prime}}(x)+\left(1-\varphi_{0}(x)\right) \max _{i=1, \cdots, a} h_{k+i}(x)-\max _{i=1, \cdots, a} h_{k+i}(x) \sum^{a} \hat{\varphi}_{i}(x) \\
& =\max _{i=1, \cdots, a} h_{k+i}(x)-\left(\hat{\varphi}_{0}(x)+\sum_{i=1}^{a} \hat{\varphi}_{i}(x)\right) \max _{i=1, \cdots, a} h_{k+i}(x)=0,
\end{aligned}
$$

and by the same argument $g(x) \geqq 0$ for $x$ in $\left\{x: \varphi_{0}(x)=0\right\}$. Therefore,

$$
\int \sum_{i=1}^{a}\left(\varphi_{i}(x)-\hat{\varphi}_{i}(x)\right) h_{k \rightarrow i}(x) d \mu(x) \geqq \begin{gathered}
M_{0} \\
k
\end{gathered} \int\left(\hat{\varphi}_{0}(x)-\varphi_{0}(x)\right) \sum_{i^{\prime}=1}^{k} h_{i^{\prime}}(x) d \mu(x) \geqq 0 .
$$

The proof is thus completed.

We shall cite the following definitions for the present section.

Definition 1. A decision function $\varphi(x)=\left(\varphi_{0}(x), \varphi_{1}(x), \cdots, \varphi_{a}(x)\right)$ is of size $\alpha$ for $H_{0}$ if

$$
E_{i} \varphi_{0}(X) \geqq 1-\alpha \quad \text { for } \quad i=1,2, \cdots, k .
$$

It is of exact size $\alpha$ for $H_{0}$ if the equality holds for all $i=1, \cdots, k$.

DEFINITION 2. $\varphi(x)$ is symmetric in power for $H_{i}$ if

$$
E_{k+1} \varphi_{1}(X)=E_{k+2} \varphi_{2}(X)=\cdots=E_{k+a} \varphi_{a}(X) .
$$

The common value of (4.9) is called the power of $\varphi$ for $H_{i} . \varphi(x)$ is called the most powerful symmetric of size $\alpha$ (MPSS $\alpha$ ) for $H_{i}$, if it maximizes each term of (4.9) subject to (4.8) and (4.9).

Now, we assume that there is a measurable transformation group $G$ on $\mathfrak{X}$ such that $G$ is isomorphic to the permutation group $\Pi=\Pi_{K} \otimes \Pi_{A}$, where $\Pi_{K}$ and $\Pi_{A}$ are the permutation groups on $K=(1,2, \cdots, k)$ or its subgroup transitive on $K=(1, \cdots, k)$ and on $A=(1,2, \cdots, a)$ or its subgroup transitive on $A=(1, \cdots, a)$, respectively. We further assume that $\mu(A)=\mu(g A)$ for all $A \in \mathfrak{A}$ and all $g \in G, h_{i}(x)=h_{\pi_{K g} i}(g x)$ for all $i=1, \cdots, k$, and all $g \in G$, and $h_{k+i}(x)=h_{k+\pi_{A} g}(g x)$ for all $i=1, \cdots, a$ and all $g \in G$, where $\pi_{A g} \in \Pi_{A}$ and $\pi_{K g} \in \Pi_{K}$, respectively, corresponding to $g$. Then we have the following corollary.

COROLLARY 2. There is a MPSS $\alpha$ test for testing $H_{0}$ against $H_{i}, i=1,2, \cdots, a$. It is given by the form (4.1) in Theorem 3 with $\xi(x)$ a constant and $\eta_{j}(x)=\frac{1-\varphi_{0}(x)}{N(x)}$, where $N(x)$ is the number of times that $\max _{i=1, \cdots, a} h_{k+i}(x)$ is attained. i.e.,

$$
\begin{aligned}
& \varphi_{0}(x)=1, \xi, 0 \text { when } \max _{i=1, \cdots, a} h_{k+i}(x)<,=,>M_{0} \cdot \frac{1}{k} \sum_{i^{\prime}=1}^{k} h_{i^{\prime}}(x) \\
& \varphi_{j}(x)=\frac{1-\varphi_{0}(x)}{N(x)}, 0 \text { when } h_{k+j}(x)=,<\max _{i=1, \cdots, a} h_{k+i}(x) .
\end{aligned}
$$

Proof. Let

$$
P_{i}\left[\left(\max _{i=1, \cdots, a} h_{k+i}(x) / k_{k}^{1} \sum_{i^{\prime}=1}^{k} h_{i^{\prime}}(x)\right) \leqq M\right]=\alpha_{i}(M) .
$$

The probability is computed under $P_{i}(i=1,2, \cdots, k)$. We shall verify that $\alpha_{i}(M)$ is independent of $i$, i. e. $\alpha_{i}(M)=\alpha(M)$ for $i=1,2, \cdots, k$. To see this, let

$$
\begin{aligned}
& \alpha_{i}(M)=P_{i}\left[\left(\max _{i=1, \cdots, a} h_{k+i}(x) /-{ }_{k}^{1} \sum_{i^{\prime}=1}^{k} h_{i^{\prime}}(x)\right) \leqq M\right]
\end{aligned}
$$

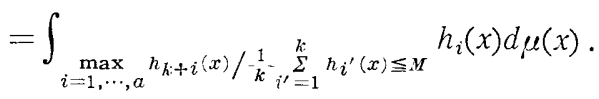


By letting $y=g x$, this is equal to

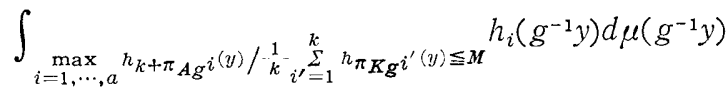

$$
\begin{aligned}
& =\int_{i=1, \cdots, a} \max _{k+\pi_{A g^{i}}(y) /-_{k}^{1}-{ }_{i^{\prime}=1}^{N} h_{\pi_{K g}}^{k} i^{\prime}(y) \leqq M} h_{\pi_{K g} i}(y) d \mu(y) \\
& =\int \max _{i=1, \cdots, a} h_{k+i}(y) / \stackrel{1}{k}^{1} \underset{i^{\prime}=1}{k} h_{i^{\prime}(y) \leqq M}^{k} h_{i^{\prime \prime}}(y) d \mu(y) \\
& =\alpha_{i^{\prime \prime}}(M), \quad i^{\prime \prime} \in(1,2, \cdots, k) .
\end{aligned}
$$

Therefore $\alpha_{i}(M)=\alpha(M)$ for $i=1,2, \cdots, k$.

Since $\alpha(M)$ is upper semi-continuous and monotonically increasing

$$
P_{i}\left[\left(\max _{i=1, \cdots, a} h_{k+i}(x) / \frac{1}{k} \sum_{i^{\prime}=1}^{k} h_{i^{\prime}}(x)\right)=M\right]=\alpha(M)-\alpha(M-0) .
$$

For any $\alpha: 0<\alpha<1$, there exists an $M_{0}$ such that $\alpha\left(M_{0}\right) \leqq \alpha \leqq \alpha\left(M_{0}-0\right)$. Put $\xi=\left[(1-\alpha)-\alpha\left(M_{0}-0\right)\right] /\left[\alpha\left(M_{0}\right)-\alpha\left(M_{0}-0\right)\right]$, and consider the decision function $\varphi(x)$ defined by (4.10). The size of $\varphi(x)$ for $H_{0}$ is

$$
E_{i} \varphi_{0}(X)=\alpha\left(M_{0}-0\right)+\frac{(1-\alpha)-\alpha\left(M_{0}-0\right)}{\alpha\left(M_{0}\right)-\alpha\left(M_{0}-0\right)}\left[\alpha\left(M_{0}\right)-\alpha\left(M_{0}-0\right)\right]=1-\alpha .
$$

We note that the $\varphi(X)$ is invariant, and hence

$$
E_{k+1} \varphi_{1}(X)=\cdots=E_{k+a} \varphi_{a}(X) .
$$

By (ii) of Theorem $1, \varphi(x)$ is obvious a MPSS $\alpha$ test for $H_{0}$ against $H_{i}(i=1,2, \cdots, a)$.

\section{§5. Remark.}

We can consider a special case when $k=a$, in this case $\pi$ is assumed to be the permutation group on $(1,2, \cdots, a)$ or its subgroup transitive on $(1,2, \cdots, a)$. Theorem and corollary above also hold in this case when $\pi_{g} i=\pi_{K_{g}} i=\pi_{A g} i$ for all $i=1, \cdots, k$.

\section{§6. Acknowlegements.}

The author is deeply indebted to Professor T. Kitagawa and Professor A. Kudô for their valuable suggestions and guidances. The author is now at Kyushu University on leave of absence from the Department of Mathematics, Tamkang College of Arts and Sciences, Taipei, Taiwan China.

\section{Kyushu University and Tamkang \\ College of Arts and Sciences}

\section{References}

[1] Doornbos, R. and Prins, H. J. (1958). On slippage tests. Indagationes Mathematicae 29, 38-55.

[2] Hall, I. J. and Kudô, A. On slippage tests-(I) A generalization of Neyman Pearson's lemma. Iowa State University (1968), submitted to Annals of Mathematical Statistics. 
[3] Hall, I. J., Kudô, A. and Yeh, N.C. On slippage tests-(II) Similar slippage tests. Iowa State University and Kyushu University (1968), submitted to Annals of Mathematical Statistics.

[4] Karlin, S. and Traux, D. R. (1960). Slippage problems. Annals of Mathematical Statistics 31, 296-324.

[5] Kudô, A. (1956a). On the testing of outlying observations. Sankaya 17, 67-76.

[6] Kudô, A. (1956b). On the invariant multiple decision procedures. Bulletin of Mathematical Statistics 6, 57-68.

[7] Mostellar, F. (1948). A k-sample slippage test for an extreme population. Annals of Mathematical Statistics 19, 58-65.

[8] Lehmann, E.L. (1959). Testing statistical hypotheses. New York, John Wiley and Sons, Inc.

[9] Paulson, E. (1952). An optimum solution to the $k$-sample slippage problem for the normal distribution. Annals of Mathematical Statistics 23, 610-616.

[10] Traux, D. R. (1953). An optimum slippage test for the variance of $k$ normal distributions. Annals of Mathematical Statistics 24, 669-674. 